Follikuläres Lymphom: Länger leben in allen Linien

Die ersten 10 Jahre MabThera ${ }^{\circledR}$ : Jetzt geht's ums Überleben!

Mit der Zulassung im Jahr 1998 war MabThera ${ }^{\circledR}$ die erste Neueinführung eines Medikaments zur Therapie der Non-Hodgkin-Lymphome seit über 12 Jahren. Bis zu diesem Zeitpunkt war die Diagnose «Lymphom» für viele Patienten ein sicheres Todesurteil. Durch den Einsatz von MabThera ${ }^{\circledR}$ das in Kombination mit einer Chemotherapie sowie als anschließende Erhaltungstherapie verabreicht wird, hat sich seitdem die Prognose vieler Patienten mit NHL deutlich verbessert.

Aggressive Lymphome: Bessere Heilungschancen Der weltweit anerkannte Lymphomexperte Professor Dr. Michael Pfreundschuh von der Universität Homburg/Saar unterstrich beim 49th Annual Meeting of the American Society of Hematology, das vom 8.-11.12.2007 in Atlanta/ Georgia stattfand, die Bedeutung der Immunchemotherapie mit MabThera ${ }^{\circledR}$ : «Rituximab ist beim aggressiven Lymphom der etablierte Standard und hat bei allen B-Zell-Lymphomen, bei denen er bisher geprüft wurde, eine erhebliche Verbesserung der Therapieergebnisse gebracht.» Große randomisierte Studien haben gezeigt, dass durch die Kombination mit MabThera ${ }^{\circledR}$ mehr Patienten geheilt werden als durch Chemotherapie allein [1-5]. In einer Studie konnte durch die Kombination mit MabThera ${ }^{\circledR}$ ein 3-JahresGesamtüberleben von $78 \%$ erzielt werden - das sind die besten Ergebnisse, die bei älteren Patienten mit aggressivem NHL je erreicht wurden [4]. Wie Pfreundschuh auf dem ASH anhand eigener Daten aus einer neuen Studie berichtete, könnten die Heilungsraten bei Patienten mit aggressivem Lymphom und schlechter Prognose in Zukunft durch ein dosisdichtes Regime mit MabThera ${ }^{\circledR}$ noch weiter steigen [6].
Auch in der Behandlung follikulärer Lymphome ist MabThera ${ }^{\circledR}$ nicht mehr wegzudenken, wie der Leiter der Deutschen Studiengruppe für niedrigmaligne Lymphome (GLSG), Professor Dr. Wolfgang Hiddemann von der Universität München, betonte: «In den letzten 10 Jahren hat sich bei der Behandlung von Patienten mit follikulären Lymphomen sehr viel getan - alle großen randomisierten Studien [7-10] haben klar belegt, dass die Verlängerung des Gesamtüberlebens bei diesen Patienten Rituximab zu verdanken ist.» Auf dem ASH wurden Daten einer neuen Metaanalyse vorgestellt, die belegen, dass das Sterberisiko durch eine Erhaltungstherapie mit MabThera ${ }^{\circledR}$ halbiert wird [11]. Fast alle Patienten, die MabThera ${ }^{\circledR}$ als Induktionstherapie erhalten, sprechen auf die Erhaltungstherapie an. Kehrt die Krankheit zurück, dann kann sie in vielen Fällen mit MabThera ${ }^{\circledR}$ wieder zurückgedrängt werden. Hatten solche Patienten vor der Einführung von MabThera ${ }^{\circledR}$ eine mittlere Überlebenserwartung von 5-10 Jahren, ist das follikuläre Lymphom mittlerweile $\mathrm{zu}$ einer chronischen Erkrankung geworden. «Rituximab plus Chemotherapie zur Remissionsinduktion, dann Rituximab als Monotherapeutikum zur Remissionserhaltung - das ist heute der absolute Standard bei der Therapie des follikuären Lymphoms», so Hiddemann. Es zeigt sich, dass auch längst Standard gewordene Therapien durch intelligente Konzepte, wie beispielsweise die Erhaltungstherapie, durchaus noch effizienter werden können.

\section{Quellen}

1 Coiffier B, et al.: Long-term results of the GELA study comparing R-CHOP and CHOP chemotherapy in older patients with diffuse large B-cell lymphoma shows good survival in poor-risk patients [abstract]. Proc ASCO 2007;25:443a.

2. Habermann T, et al.: Rituximab-CHOP versus $\mathrm{CHOP}$ alone or with maintenance rituximab in older patients with diffuse large B-cell lymphoma. J Clin Oncol 2006; 24: 3121-3127.

3. Pfreundschuh M, et al.: CHOP-like chemotherapy plus rituximab versus CHOP-like chemotherapy alone in young patients with good-prognosis diffuse large B-cell lymphoma: A randomised controlled trial by the MabThera International Trial (MinT) Group. Lancet Oncol 2006;7:379-389.

4. Pfreundschuh M, et al.: 6 vs. 8 cycles of biweekly CHOP-14 with or without rituximab for elderly patients with diffuse large B-cell lymphoma (DLBCL): results of the completed RICOVER-60 trial of the DSHNHL [abstr]. Blood 2006;107:205

5. Sehn LH, et al.: Introduction of combined CHOP plus rituximab therapy dramatically improved outcome of diffuse large B-cell lymphoma in British Columbia. J Clin Oncol 2005: 23: 5027-5033.

6. Pfreundschuh M, et al: ASH 2007, Abstract 789. 7. Marcus R, et al.: CVP chemotherapy plus rituximab compared with CVP as first-line treatment for advanced follicular lymphoma. Blood 2005;105: 1417-1423.

8. Hiddemann W, et al.: Frontline therapy with rituximab added to the combination of cyclophosphamide, doxorubicin, vincristine, and prednisone (CHOP) significantly improves the outcome for patients with advanced-stage follicular lymphoma compared with therapy with $\mathrm{CHOP}$ alone: results of a prospective randomized study of the German Low-Grade Lymphoma Study Group. Blood 2005;106:3725-3732.

9. Herold M, et al.: Rituximab added to first-line mitoxantrone, chlorambucil, and prednisolone chemotherapy followed by interferon maintenance prolongs survival in patients with advanced follicular lymphoma: an East German Study Group Hematology and Oncology Study. J Clin Oncol 2007; 25: 1986-1992. 10. Salles G, et al.: Rituximab added to alpha IFN+ CHVP improves the outcome of follicular lymphoma patients with a high tumor burden: First analysis of the GELA-GOELAMS FL-2000 randomized trial in 359 patients [abstr]. Blood 2004;104:160a and Udate (Final Analysis): Salles G, et al.: ASH 2007, Abstr 792

11. Vidal L, et al.: ASH 2007, Abstract 3408.

Roche Pharma AG

Herr Dr. H.-U. Jelitto

Emil-Barell-Straße 1, 79639 Grenzach-Wyhlen

Tel. +49 7624 14-2400, Fax -3366

www.roche.de

\title{
PharmaTicker+++ PharmaTicker+++ PharmaTicker+++ PharmaTicker+++
}

Celgene GmbH. Lenalidomid (Revlimid $\left.{ }^{\circledR}\right)$ gehört zur neuen Wirkstoffgruppe der immunmodulatorischen Substanzen $\left(\mathrm{IMiDs}^{\circledR}\right)$ und ist in Europa der Schweiz und den USA für eine Kombinationstherapie mit Dexamethason bei Patienten mit einem multiplen Myelom zugelassen, die bereits eine Standardtherapie erhalten haben. Der Wirkstoff wird auch bei anderen hämato-onkologischen Erkrankungen wie chronisch lymphatischer Leukämie und Non-Hodgkin-Lymphomen untersucht.

Weber Shandwick Frankfurt

Kristina Natter

Tel. +49 69 913043-10

KNatter@webershandwick.com
Essex Pharma GmbH. Pegyliert liposomales Doxorubicin $\left(\mathrm{CAELYX}^{\circledR}\right)$ hat im Dezember 2007 die Zulassung zur Behandlung des progressiven multiplen Myeloms in Kombination mit dem Proteasom-Inhibitor Bortezomib erhalten. Diese Kombinationstherapie hatte sich in einer großen randomisierten Studie gegenüber einer Bortezomib-Monotherapie als überlegen erwiesen.

Weitere Informationen bei Medizin und Markt GmbH Franziska Wagenschwanz Tel. +49 89 3-8393022, Fax -39075 wagenschwanz@medizin-und-markt.de
Bayer HealthCare AG. Die Europäische Kommission hat die Marktzulassung von MabCampath ${ }^{\circledR}$ (Alemtuzumab) für die Behandlung von Patienten mit chronischer lymphatischer Leukämie vom B-Zell-Typ (B-CLL) erteilt, für die eine Fludarabin-Kombinationstherapie unangemessen ist. MabCampath ist der erste und einzige in Europa für die Behandlung der B-CLL zugelassene monoklonale Antikörper.

Bayer HealthCare AG

Unternehmenskommunikation

Anna Schuberth

Tel. +493046815942

anna.schuberth@bayerhealthcare.com 
Studie im Journal of Clinical Oncology

\section{UFT $^{\circledR}$ (Tegafur Uracil) zeigt auch bei älteren Patienten gute Wirksamkeit und Verträglichkeit}

Das orale Fluoropyrimidin UFT ist in Kombination mit Calciumfolinat auch in der Therapie von älteren Patienten ( $\geq 75$ Jahre) mit kolorektalem Karzinom wirksam. Darüber hinaus wird UFT in dieser Patientenpopulation gut vertragen, vor allem im Vergleich mit anderen Fluoropyrimidinregimen. Das belegen die Ergebnisse einer Phase-II-Studie, die kürzlich im Journal of Clinical Oncology veröffentlicht wurde [1].

In die multizentrische Studie der Eastern Cooperative Oncology Group wurden 55 nicht vorbehandelte Patienten über 75 Jahre mit metastasiertem kolorektalem Karzinom eingeschlossen. Die Patienten erhielten UFT $300 \mathrm{mg} / \mathrm{m}^{2}$ pro Tag in drei separaten Dosen alle 8 Stunden in Kombination mit je $30 \mathrm{mg}$ Calciumfolinat für 28 Tage, gefolgt von einer 7-tägigen Pause. Dieser Zyklus wurde alle 35 Tage bis zur Progression wiederholt. Primärer Endpunkt war das Ansprechen. Zusätzlich wurden das mediane Gesamtüberleben, das mediane progressionsfreie Überleben und die Verträglichkeit der Kombinationstherapie untersucht.

Die Ansprechrate betrug 22\%. Das mediane Gesamtüberleben lag bei 13 Monaten und das mediane progressionsfreie Überleben bei 4,6 Monaten. Diese Ergebnisse sind vergleichbar mit Daten aus anderen Studien mit UFT oder anderen Fluoropyrimidin-basierten Regimen, unabhängig von der Altersgruppe.

Dabei konnte gezeigt werden, dass das gute $\mathrm{Ne}$ benwirkungsprofil von UFT auch auf ältere Patienten übertragbar ist. Die häufigste Grad-3/4Toxizität war Diarrhö. Die Diarrhö-Episoden waren gut behandelbar und führten nicht zu einem vorzeitigen Therapieabbruch. Neutropenie trat nur in geringem Maße auf $(5,4 \%)$. Außer- dem wurde kein Grad-3/4-Hand-Fuß-Syndrom unter der UFT-Therapie beobachtet. Dies ist von besonderer Bedeutung, da die bei anderen Fluoropyrimidin-basierten Chemotherapien häufige Nebenwirkung die Lebensqualität der Patienten beeinflusst. So kann das Auftreten eines HandFuß-Syndroms zu einer starken Beeinträchtigung des Alltagslebens sowie zur Immobilität des Patienten führen.

Die Autoren schlussfolgern, dass die Ergebnisse dieser einarmigen, offenen Studie konsistent seien mit den bisherigen Daten zur Wirksamkeit und Sicherheit von UFT in Kombination mit Calciumfolinat bei der Therapie des kolorektalen Karzinoms. UFT könne auch bei Patienten über 75 Jahre eingesetzt werden. Es biete eine vergleichbare Wirksamkeit wie andere Fluoropyrimidin-basierte Chemotherapien bei geringerer Toxizität als bei Bolus-Gabe von 5-FU oder Capecitabine, das mit einem merklichen Auftreten des Hand-Fuß-Syndroms assoziiert sei.

«Mit UFT steht eine orale Alternative zu der infusionalen Gabe von 5-FU zur Verfügung, mit vergleichbarer Wirksamkeit. Zudem ist das geringe Auftreten des Hand-Fuß-Syndroms ein besonderer Vorteil», kommentierte PD Dr. med Ralf-Dieter Hofheinz vom Universitätsklinikum Mannheim. «Auch viele Patienten bevorzugen die orale Verabreichung der Therapie. Die gute Verträglichkeit sowie der geringere Zeitaufwand tragen ebenso zur Lebensqualität bei wie der Verzicht auf Pumpen oder Ports. Das wiederum erhöht die Compliance der Patienten. Und eine regelmäßige Einnahme ist schließlich eine wichtige Voraussetzung für den Therapieerfolg.»

Die Ergebnisse dieser Studie sind besonders relevant für den klinischen Alltag. 60-70\% der Patienten, bei denen ein Tumor diagnostiziert wird, sind über 65 Jahre alt. Dennoch ist diese wichtige Patientenpopulation in klinischen Studien häufig unterrepräsentiert. Aus Gründen der Eignung und Selektion liegt der Altersdurch- schnitt von Patienten in klinischen Studien beim kolorektalen Karzinom bei rund 60 Jahren.

Fluoropyrimidin-basierte Therapieregime bilden das Rückgrat in der Behandlung des kolorektalen Karzinoms, sowohl in der adjuvanten als auch in der metastasierten Situation. UFT ist eine 1:4Kombination aus Tegafur und Uracil. Tegafur ist ein sogenanntes Prodrug von 5-Fluorouracil und wird im Körper fast vollständig dazu umgewandelt. Uracil hemmt die Dihydropyrimidin-Dehydrogenase, das für die Metabolisierung und Inaktivierung von 5-FU verantwortliche Enzym. In der EU ist UFT seit 2002 in Kombination mit Folinsäure für die Erstlinientherapie des metastasierten kolorektalen Karzinoms zugelassen. Diese Zulassung beruht auf zwei randomisierten Phase-III-Studien, in denen UFT eine vergleichbare Wirksamkeit wie die Bolus-Gabe von Fluoropyrimidin gezeigt hat $[2,3]$. Darüber hinaus war die Toxizität unter der oralen Applikation geringer als bei der Bolus-Gabe.

\section{Referenzen}

1 Hoechster HS, et al.: Phase II study of uracil-tegafur with leucovorin in elderly ( $\geq 75$ years old) patients with colorectal cancer: ECOG 1299. J Clin Oncol 2007;25:5397-5402.

2 Douillard JY, et al.: Multicenter phase III study of uracil/tegafur and oral leucovorin versus parenteral fluorouracil and leucovorin in patients with previously untreated metastatic colorectal cancer.

J Clin Oncol 2002;20:3605-3616.

3 Carmichael J, et al.: Randomized comparative study of tegafur/uracil and oral leucovorin versus parenteral fluorouracil and leucovorin in patients with previously untreated metastatic colorectal cancer. J Clin Oncol 2002;20:3617-3627.

IntraMedic $\mathrm{GmbH}$

Yvonne Söltl

Dornhofstraße 34

63263 Neu-Isenburg

Tel. +49 6102 7993-345, Fax -301

Yvonne_Soeltl@yr.com

\section{PharmaTicker+++ PharmaTicker+++ PharmaTicker+++ PharmaTicker+++}

AstraZeneca GmbH. Beim San Antonio Breast Cancer Symposium (13.-16.12.2007) wurden neue Daten der ATAC-Studie (Anastrozol Tamoxifen alone or in Combination) präsentiert. Die Auswertung nach einem medianen Follow-Up von 100 Monaten bestätigte die Ergebnisse der vorangegangenen Analysen: Anastrozol (Arimidex ${ }^{\circledR}$ ) reduziert das Rezidivrisiko signifikant und verlängert das krankheitsfreie Überleben.

Publicis Vital PR

Frau Natalie Fiebig

Hansaring 97, 50670 Köln

Tel. +49 $221912719-85$

natalie.fiebig@ publicis-vital-pr.de
Roche Pharma AG. Ibandronat (Bondronat ${ }^{\circledR}$ ) ist zur Prävention skelettaler Komplikationen sowie zur Schmerzreduktion auch in der klinischen Praxis wirksam und zeigt ein günstiges renales Verträglichkeitsprofil. Dieses vorläufige Ergebnis einer aktuell durchgeführten Untersuchung wurde im Rahmen des San Antonio Breast Cancer Symposiums (13.-16.12.2007) vorgestellt. Roche Pharma AG Herrn Dr. H.-U. Jelitto Emil-Barell-Straße 1 79639 Grenzach-Wyhlen Tel. +49 7624 14-3715, Fax -3366 www.roche.de
GlaxoSmithKline GmbH. Präklinische und erste klinische Hinweise lassen vermuten, dass die Kombinationstherapie mit der antiangiogenetischen Substanz Pazopanib und dem dualen ErbB1/ErbB2-Hemmstoff Lapatinib das Tumorwachstum stärker hemmt als jedes der Mittel allein. Für eine multizentrische, placebokontrollierte, randomisierte, doppelblinde Studie der Phase III werden für Deutschland noch Studienteilnehmerinnen gesucht.

GlaxoSmithKline GmbH \& Co. KG Arnd Prilipp

Tel. +49 89 36044-8117, Fax -8066 arnd.prilipp@gsk.com 